\title{
Digital Control System Analysis and Design*
}

\author{
Charles L. Phillips and H. Troy Nagle, Jr.
}

Reviewer: R. P. OFFEREINS

Department of Electrical Engineering. Twente University of Technology, P.O. Box 217, Enschede, The Netherlands.

THE воок Digital Control System Analysis and Design by Charles L. Phillips and $\mathrm{H}$. Troy Nagle, $\mathrm{Jr}$. is a good and modern book on discrete-time systems and its microprocessor implementations. Its 550 pages contain nearly all that a control engineer needs in practice. The classical approach using $z$-transforms, bilinear $w$ transforms and digital transfer functions as well as the effects of sampling and reconstruction when entering or leaving the computer by ADC or DAC are dealt with in sufficient detail.

These methods already described in the "old" books of Jury, Rassazini. Kuo, Tou and others have lost nothing of their usefulness. But also the "modern" approach using state-space description methods for linear optimal control and state estimation by observers is not forgotten. About 350 pages of the book are devoted to the subjects mentioned so far. The remaining part of the book deals with digital filters and methods to implement these filters with microprocessors. Effects of quantization and signal scaling with the influence of the chosen filter structure on the accuracy due to these effects are described. The structure of many microprocessors is given and their performance is compared. Many examples of programmes in assembly language are given. The Appendix contains a number of computer programs written in BASIC.

The book hardly contains anything about stochastic signals, which is a pity. A little extension describing the relation between autocorrelation functions, power density spectra and variance of discrete-time signals would have been useful and of practical importance at various points as will be shown here after. The following part of this review will deal with various specific points.

The book introduces the bilinear $w$-transform as

or

$$
\begin{aligned}
& w=(T / 2) *(z-1) /(z+1) \\
& w=(T / 2) * t \operatorname{sh}(s * T / 2) .
\end{aligned}
$$

The extra factor $T / 2$ is not used in other textbooks. The usefulness of this transform for frequency response methods becomes more clear in this way as $w$ approaches $s$ for $T$ going to 0 .

This book, as in all books on the subject introduces the Laplace transform of a discrete-time signal, which actually is a sequence of equally spaced numbers having no Laplace transform, by replacing each number with magnitude $f$ by a pulse with magnitude ( = area) equal to $f$. It has advantages to take this magnitude equal to $f * T$ as is illustrated below. Formulas describing signals before and after sampling become the same when $T$ approaches 0 . The dimensions of signals before and after sampling are the same. This is also true before and after reconstruction. The frequency spectrum of the signal after sampling can simply be obtained by adding the periodically repeated spectra of the signal before sampling without an extra factor $1 / T$ and reconstruction filters have magnitude 1 for zero frequency. Also the autocorrelation function and the correspond-

* Digital Control System Analysis and Design by C. L. Phillips and H. Troy Nagle, Jr. Published by Prentice-Hall, Englewood Cliffs, New Jersey (1984), US $\$ 49.90$

The books which are reviewed in the IF AC-Journal Automatica are not necessarily endorsed by IFAC, the editors, or the publisher nor are the reviewers' opinions or comments about the books. ing power density spectrum for a sampled signal can be introduced as the sampled autocorrelation function belonging to the signal before sampling without confusing factors $T$. The important quantity mean square value of a number sequence then equals the area below one period of the power density spectrum. However the autocorrelation function and the power density spectrum do not occur at all in the book.

The book discusses the zero-order hold, the first-order hold and the fractional-order hold; the latter as a weighted sum of the first two. The first one is the "normal" digital-analog converter and the last two require a linear slope of the reconstructed signal, which is a more complicated device. However the first-order hold gives a more accurate reconstruction as can be seen from the fact that the velocity constant for the zero-order hold equals $T / 2$ and for the first-order hold it is infinite. Apart from this accuracy an important point for reconstruction is the ripple in the output which can be considered as the remnants of the frequency band around the sampling frequency and the area underneath this band of the power density spectrum is a measure for the mean square value. This point is neglected in the book. It is an example of the importance of stochastic signals. Too much ripple in control signals may be harmful: for example servo motors could be heated, amplifiers could be saturated and mechanical wear may occur

A good "linear slope" reconstruction element, that I have not seen in any book is also not mentioned in this book. It consists of an interpolation between the present value and the value that is first-order extrapolated. Thus sudden step changes at the sampling times are avoided and the ripple reduction is better, while still an infinite velocity constant remains. The acceleration constant is somewhat larger than for the first-order hold.

The derivation in the book of the full-order observer leads to the error equation

$$
e(k+1)=(A-G C) * e(k)+B * w(k)-G * v(k)
$$

where $w(k)$ and $v(k)$ are introduced as plant disturbance vector and measurement inaccuracies. This equation is derived using the design criterion that the system input affects the plant state and the observer state in the same manner. The choice of $G$ is still free and can be chosen as is done in the book by choosing a characteristic equation.

A small extension could have given the link to the Kalman filter equations. A least squares estimation together with a notion of stochastic signals would have done the job. The time constants of the observer are suggested in the book to be chosen about three times the values of those of the control loop controlling the plant, which is very reasonable. The Kalman approach would have improved the motivation for the bandwidth of the observer from the viewpoint of a compromise between a high value necessary to follow the plant state variations due to unknown plant influences and a low value necessary for removing measurement uncertainties

The book pays much attention to digital filters. However, the discrete Fourier transform, which of course is given already in the beginning of the book, is not considered as a digital filter though it is of much practical importance, especially the fast algorithm known as Fast Fourier Transform for a number of points $N$ equal to a power of 2 . In fact such a transform constitutes a number of narrow band filters in parallel. It would probably go too far to give this algorithm in the book. However the frequency characteristics of these filters are important and can easily be derived using the complex convolution method of Appendix III 
We have to consider the product of a sinusoidal signal with a frequency spectrum consisting of two pulse functions, one for a positive frequency value and one for a negative frequency and $N$ equally spaced pulses of which the Fourier transform is easily calculated as the sum of $N$ terms $\exp (j \omega k T)$. A paragraph in the chapter about digital filters could have been devoted to this subject.

In the book the link between the actual real world of the plant to be controlled and the digital world from which this plant is controlled is in all cases such that the loop is closed via the computer. There are cases where the computer via its DAC (digital analog converter) delivers the desired value. Loops requiring a high bandwidth sometimes, if closed outside the computer, ask very short sampling times. An example of this is the tacholoop in a servo mechanism. On the other hand when the computer delivers the desired value, the accuracy of the reconstruction is more critical due to the fact that the range of the desired value generally is larger than that of the error signal. Then the "linear slope" prediction/interpolation reconstruction. suggested as a possibility above, sometimes will become necessary. Another point not mentioned in the book is the necessity of presampling filters for elimination of measurement noise. Frequency components above half of the sampling frequency should be removed. Here again there is a point where the lack of the concept of a stochastic signal in its simplest form is felt

The remarks made in this review do not diminish the appreciation of the reviewer for the book as already expressed in the introduction. The book is indeed very suitable for graduate courses and for self-study for practising engineers. Each chapter starts with an introduction and ends with a summary. Also each chapter contains a number of well-chosen examples and problems and an extensive reference list.

A second printing, which hopefully will soon be necessary could possibly include some or all of the points mentioned without making the book too voluminous. There are hardly subjects that could be omitted without reducing the value of the book.

\title{
Computer-Controlled Systems: Theory and Design*
}

\author{
Karl J. Åström and Björn Wittenmark
}

Reviewer: C. RICHARD JOHNSON, JR.

School of Electrical Engineering, Cornell University, Ithaca, NY 14853, U.S.A.

THE BOOK Computer-Controlled Systems: Theory and Design by Aström and Wittenmark is, and will be reviewed here as, a text. It is not a handbook-style reference filled with crucial formulas, tables of comparative examples, and elaborately detailed case studies. Nor is it a mathematical treatise composed about a theoretical structure of broadly generic abstraction. It is instead a text for the current university student of digital control theory and the practising control engineer educated in analog control design but without classroom training in digital control. These two categories of student-readers are advised to examine this text for the same reason: its blend of practical motivation, sound pedagogy and theoretical breadth. The compromises in topic selection and depth of coverage inherent in a text with this style are likely to be the sources of any negative criticisms, primarily by text-seeking teachers. The purpose of this review is to share one teacher's reaction to these choices and their execution.

In the preface, the authors comment that the "purpose of this book is to present control theory that is relevant to the analysis and design of computer-controlled systems, with an emphasis on basic concepts and ideas". For this purpose the authors use practical motivation primarily to justify the relevance of theoretical topics to be presented. As such, the practical issues cited determine the topic selection. For example, the second sentence of Chapter 6, entitled "Disturbance Models", states that "without disturbances there is no need for feedback control". Both deterministic and stochastic disturbance models are presented in Chapter 6 due to their proven usefulness in control design. The inclusion of stochastic models for disturbances then requires coverage of the optimal control methods in Chapters 11 and 12 to realize their usefulness.

With the emphasis on pedagogical support from sampled-data practice, the motivating applications cited, which are drawn primarily from process and servo control, are not overloaded with the myriad of details associated with real problems. Instead, their pedagogically pertinent essence is abstracted and

* Computer-Controlled Systems: Theory and Design by K. J. Aström and B. Wittenmark. Published by Prentice-Hall, Englewood Cliffs, New Jersey (1984), US \$44.50. emphasized. For example, the examples in Chapter 1 are used to demonstrate the need for and uses of computer-control theory. In Chapter 7 they illustrate design philosophies, objectives, and tradeoffis. The examples in Appendix A describe physical sources of simple mathematical models, such as the double integrator, which are used repeatedly as academic examples throughout the text. These examples in Chapters 1 and 7 and Appendix $A$ are indicative of the balance the authors seek between practice and theory. The enticement to the reader is that the practicalities of sampled-data control, which the authors do cite, are directly addressable by the theory being presented.

Another appealing pedagogical feature of this text is the printing of the intended message of each chapter just below its title. To this reviewer these goals are more succinctly revealing of the scope of the text than the table of contents. The remainder of this review uses these stated goals as a guide in discussing the choices in topic coverage made by the authors.

Chapter 1 combines motivation for the need for a theory to describe the use of a digital computer as a feedback compensator of an analog plant with a brief sketch of the history of its development. The reference list at the end of Chapter 1 is indicative of the use of references throughout the text. The authors cite, within a brief commentary, seminal and tutorial sources for the concepts just presented. The reader's ability to divert, when desired, to a more detailed presentation is greatly facilitated.

Chapter 2 discusses Shannon's sampling theorem, hold reconstruction, frequency folding due to aliasing, and sample period selection. The chapter closes with the statement that the "choice of sampling period will be discussed several times later in the book". Though they preceded this statement with a description of the most useful rule-of-thumb for sample rate selection (with relation to the desired closed-loop bandwidth), the authors are foreshadowing the use of a wide range of factors in the application-dependent art of sample rate selection. The subject of sampling and its analysis when included in a control system is examined with more mathematical detail in Chapters 3 and 4 . The authors adopt the appealing perspective of viewing the control system from the perspective of the digital controller in Chapter 3 and from the perspective of the analog plant, or the analog output actually being controlled, in Chapter 4 .

Chapter 3 focuses on the zero-order-hold discrete-time equivalent of a continuous-time state-space plant model. 